\title{
Acute Toxic Effects of Telmisartan in Spontaneously Hypertensive Rats Fed a High Fructose Diet
}

\author{
J. ŠILHAVÝ ${ }^{1}$, P. MLEJNEK ${ }^{1}$, M. ŠIMÁKOVÁ ${ }^{1}$, I. VANĚČKOVÁ ${ }^{1}$, M. BEHULIAK ${ }^{1}$, \\ O. KUDA ${ }^{1}$, E. STICOVÁ ${ }^{2,3}$, M. JIRSA ${ }^{2}$, M. PRAVENEC ${ }^{1}$
}

${ }^{1}$ Institute of Physiology of the Czech Academy of Sciences, Prague, Czech Republic, ${ }^{2}$ Institute for Clinical and Experimental Medicine, Prague, Czech Republic, ${ }^{3}$ Department of Pathology, Third Faculty of Medicine, Charles University, Prague, Czech Republic

Received May 30, 2018

Accepted June 28, 2018

On-line September 11, 2018

\section{Summary}

Telmisartan is an angiotensin receptor blocker (ARB) and a selective peroxisome proliferator activated receptor gamma (PPARG) modulator. Recently, we tested metabolic effects of telmisartan ( $5 \mathrm{mg} / \mathrm{kg}$ body weight) in spontaneously hypertensive rats (SHR) fed a diet containing $60 \%$ fructose, a widely used model of the metabolic syndrome. Surprisingly, we observed acute toxic effects of telmisartan. Rats lost body weight rapidly and died within 2 to 3 weeks due to bleeding into the upper gastrointestinal tract. SHR fed a high fructose diet and treated with telmisartan exhibited rapid decrease in blood pressure when compared to the SHR fed a high fructose diet and treated with valsartan. Concentrations of both unconjugated telmisartan and telmisartan glucuronide in the liver of SHR rats fed a high fructose diet were approximately 4 fold higher when compared to Brown Norway (BN) rats fed the same diet. Plasma concentrations of unconjugated telmisartan in the SHR were about 5 fold higher when compared to $\mathrm{BN}$ rats while plasma levels of telmisartan glucuronide were similar between the strains. Testing of other rat strains, diets, and the ARB valsartan showed that toxic effects of telmisartan in combination with high fructose diet are specific for the SHR. These results are consistent with the possibility that in some circumstances, SHR are predisposed to telmisartan toxicity possibly because of a genetically determined disturbance in telmisartan metabolism.

\section{Key words}

Telmisartan • Spontaneously hypertensive rat • Fructose • Toxic

\section{Corresponding author}

M. Pravenec, Institute of Physiology of the Czech Academy of Sciences, Vídeňská 1083, 14220 Prague, Czech Republic. E-mail: pravenec@biomed.cas.cz

\section{Introduction}

The spontaneously hypertensive rat (SHR) is the most widely studied model of essential hypertension and under specific environmental condition, such as when fed a diet with high content of fructose, it also develops disorders of lipid and glucose metabolism that are characteristic for metabolic syndrome (Pravenec et al. 2014). Telmisartan is an angiotensin receptor blocker (ARB) with unique chemical properties that enable it to partially activate the peroxisome proliferator activated receptor gamma (PPARG) and thus ameliorate insulin resistance (Benson et al. 2004). Recently, we tested metabolic effects of telmisartan using a clinically relevant dose of telmisartan in the drinking water. Surprisingly, SHR rats showed severe loss of body weight and all died within 2 to 3 weeks. In the current study, we investigated the predisposition of SHR to these acute toxic effects of telmisartan.

\section{Methods}

Animals

We used 3-month-old male rats from the SHR/OlaIpcr (referred to as SHR) and BN-Lx/Cub 
(referred to as $\mathrm{BN}$ ) inbred strains, and from Wistar outbred stock. All rats were obtained from the Institute of Physiology of the Czech Academy of Sciences, Prague. Rats were kept at a 12/12 h light/dark period with free access to standard laboratory chow or a diet with $60 \%$ fructose (K4102.0 diet, Hope Farms, The Netherlands) and water. The experiments were performed in agreement with the Animal Protection Law of the Czech Republic and were approved by the Ethics Committee of the Institute of Physiology of the Czech Academy of Sciences, Prague.

\section{Experimental protocol}

SHR and Wistar male rats were divided into 4 groups: i) rats fed a standard chow and tap water, ii) rats fed a standard chow and treated with telmisartan in tap water, iii) rats fed a high fructose diet and tap water, and iv) rats fed a high fructose diet and treated with telmisartan in tap water ( $\mathrm{N}=6$ rats per each group). We added telmisartan to the drinking water in an amount that provided a telmisartan dose of approximately $5 \mathrm{mg} / \mathrm{kg}$ body weight of telmisartan per day. Telmisartan was added to the drinking water after solubilization in a small amount of ethanol. Drinking water of control groups contained the same miniscule amount of ethanol without telmisartan. BN rats were tested only when treated with telmisartan and fed a high fructose diet. In addition, a separate group of SHR rats was treated with valsartan ( $5 \mathrm{mg} / \mathrm{kg}$ body weight in tap water) for 3 weeks.

\section{Biochemical analyses}

Sera for biochemical analyses were collected from $\mathrm{BN}$ rats treated with telmisartan and fructose, and from the separate groups of SHR (i-iv as above) on day 13 of treatment. Biochemical parameters in urine and sera were performed in clinical laboratories of the Institute for Clinical and Experimental Medicine in Prague.

Telmisartan and telmisartan acyl- $\beta$-Dglucuronide were purchased from Santa Cruz Biotechnology (CA, USA). All solvents and chemicals were of MS grade, bought from Sigma Aldrich. For quantitation of telmisartan and its glucuronide conjugate, liver samples $(\sim 50 \mathrm{mg})$ were homogenized in $0.5 \mathrm{ml}$ of methanol and $0.5 \mathrm{ml}$ of acetonitrile using a bead mill MM400 (Retsch, Germany), centrifuged $12,000 \mathrm{~g}$ at $4{ }^{\circ} \mathrm{C}$ for $10 \mathrm{~min}$, supernatants filtered and stored at $-80{ }^{\circ} \mathrm{C}$ until LC-MS/MS analysis. Differential ion mobility mass spectrometry system SelexION/QTRAP 5500 (Sciex,
USA) coupled to UPLC Dionex 3000 RSLC (Thermo, USA) was used to quantify analytes. Telmisartan and telmisartan glucuronide were separated using C8 Kinetex column (50x2.1 mm, $1.7 \mu \mathrm{m}, 100 \mathrm{~A}$, Phenomenex, USA) and gradient elution as described before (Yan et al. 2008).

\section{Blood pressure measurements}

Arterial blood pressures were measured continuously by radiotelemetry (Data Sciences International, St. Paul, USA) in paired experiments in conscious, unrestrained SHR male rats ( $\mathrm{N}=8$ per group). All rats were allowed to recover for at least 7 days after surgical implantation of radiotelemetry transducers before the start of blood pressure recordings and start of treatment with either telmisartan or valsartan in drinking water in doses of $5 \mathrm{mg} / \mathrm{kg}$ body weight. Pulsatile pressures were recorded in 5-s bursts every $10 \mathrm{~min}$ throughout the day and night.

\section{Statistical analysis}

Values are expressed as means \pm SEM. Individual groups were compared by unpaired Student t-test. One-way ANOVA was used for the analysis of statistical significance of biochemical parameters. The 12-h mean values of systolic and diastolic blood pressures were analyzed by repeated measures of ANOVA with grouping effect of treatment and repeated measurements in time. Statistical significance was defined as $\mathrm{P}<0.05$.

\section{Results}

Acute toxic effects of telmisartan specific for the SHR strain

As shown in Figure 1A, the SHR fed a standard chow or high fructose diet without treatment showed similar increases in body weight. Treatment with telmisartan in the SHR fed a standard chow was associated with a small but significant decrease in body weight but without any other adverse effects. On the other hand, treatment with telmisartan in the SHR fed a high fructose diet was associated with a dramatic decrease in body weight and all rats died within 15-19 days. Combination of high fructose diet with telmisartan treatment was not associated with toxic effects in BN rats (Fig. 1A) or in Wistar rats (Fig. 1B).

To test whether the above described acute toxic effects are specific for telmisartan, we treated fructose fed SHR with another ARB valsartan. As shown in 
A.

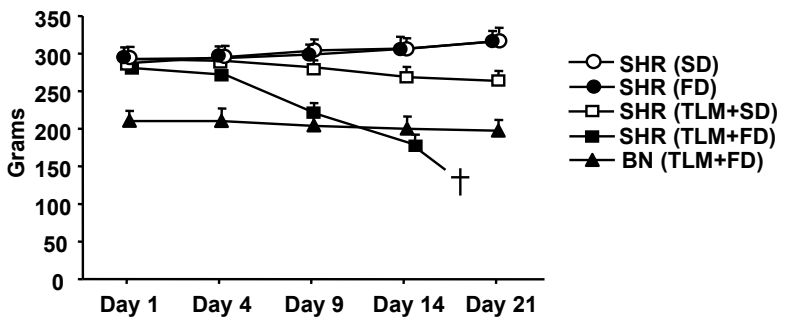

B.

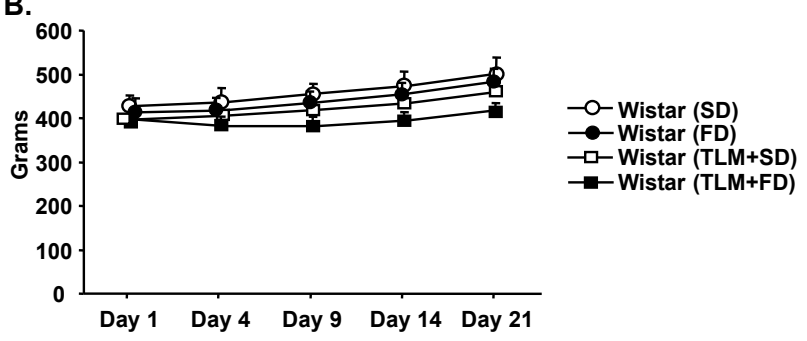

C.

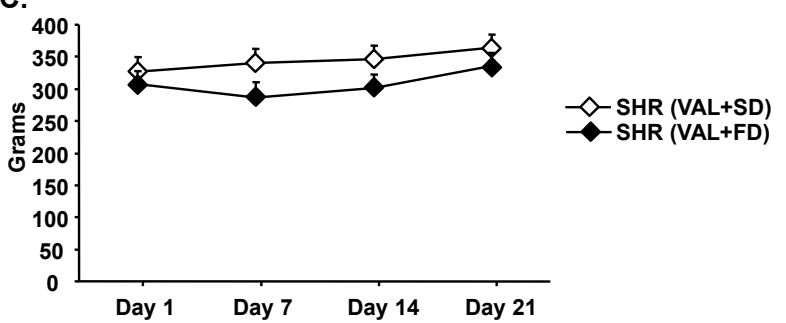

Fig. 1. Body weight in rats treated with telmisartan or valsartan and fed either standard diet or diet with $60 \%$ of fructose. A. SHR rats fed a standard or high fructose diet without telmisartan showed increase in body weight; SHR rats fed a high fructose diet and not treated with telmisartan showed a mild decrease in body weight and appeared clinically healthy; SHR rats fed a high fructose diet and treated with telmisartan showed rapid weight loss and all rats died before the end of the 3rd week; BN rats fed a high fructose diet and treated with telmisartan showed a mild decrease in body weight and appeared clinically healthy. B. Wistar rats showed increases in body weight with all combinations of diet and treatment. C. SHR rats fed a high fructose diet and treated with valsartan showed mild intermittent decrease in body weight and appeared clinically healthy. FD - fructose diet, SD - standard diet, TLM telmisartan, VAL - valsartan.

Figure 1C, we observed a small interim decrease in body weight but no other adverse effects. Taken together, these results provide evidence for acute toxicity of telmisartan in combination with the high fructose diet which was observed in the SHR strain but not in the BN strain or in non-inbred Wistar rats.

Effects of telmisartan and valsartan on blood pressure in SHR fed a high fructose diet

As shown in Figure 2, SHR rats treated with telmisartan and fed a high fructose diet showed progressively reduced blood pressures when compared to SHR rats treated with valsartan and fed the same diet.
A.

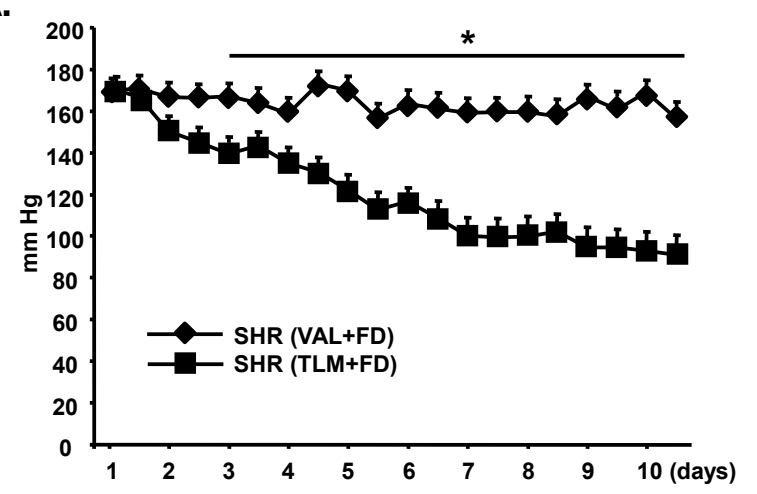

B.

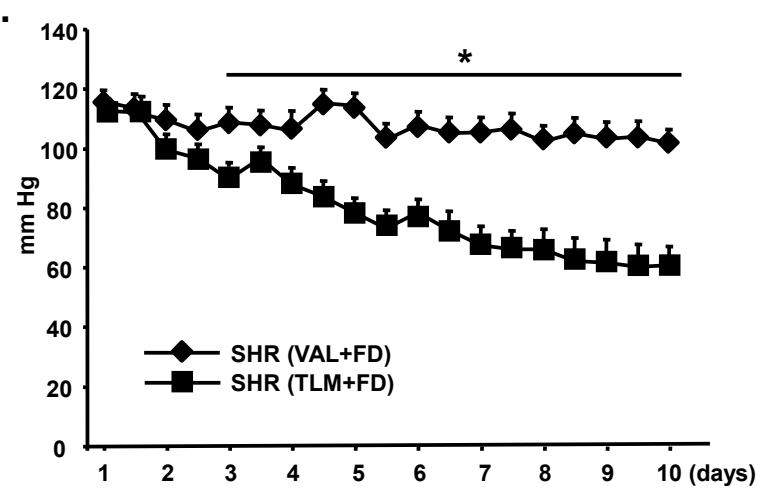

Fig. 2. Effects of high fructose diet in combination with telmisartan or valsartan on blood pressure in the SHR. Twelvehour average systolic blood pressures (A) and diastolic blood pressures (B) were significantly decreased in rats treated with telmisartan when compared to rats treated with valsartan. * denotes $\mathrm{P}<0.05$. FD - fructose diet, TLM - telmisartan, VAL valsartan.

\section{Clinical biochemistry and histopathological changes}

As shown in Table 1, SHR rats that were fed a high fructose diet and treated with telmisartan showed evidence of impaired liver and kidney function. There were no significant morphological changes observed in livers, except for mild centrolobular steatosis. Increased bilirubin levels (Table 1) were likely to be secondary to gastrointestinal bleeding, disintegration of erythrocytes and consequential absorption of bilirubin from the intestine. Acute tubular damage was observed in kidney which was likely to be secondary to hypovolemic shock associated with significant internal bleeding. In addition, rats had significantly increased serum urea and creatinine concentrations (Table 1). The most significant pathological changes were found in the upper gastrointestinal tract (esophagus and stomach) including erosions and ulceration with bleeding as well as inflammatory infiltrates with eosinophilic leukocytes (Fig. 3). 
Table 1. Clinical biochemistry in SHR rats fed standard diet (SD) and high fructose diet (FD) and treated with telmisartan (TLM) or placebo in comparison to BN rats fed high fructose diet and treated with telmisartan for 13 days.

\begin{tabular}{lccccc}
\hline Parameter & SHR & SHR & SHR & SHR & BN \\
& (SD + placebo) & (FD + placebo) & (SD + TLM) & (FD + TLM) & (FD + TLM) \\
\hline Na $(\mathrm{mmol} / \mathrm{l}) *$ & $140.66 \pm 0.33^{\#}$ & $140.25 \pm 0.56^{\#}$ & $143.35 \pm 0.85$ & $145.30 \pm 1.39$ & $138.07 \pm 0.71^{\#}$ \\
K $(\mathrm{mmol} / \mathrm{l}) *$ & $6.06 \pm 0.43^{\#}$ & $6.20 \pm 0.50^{\#}$ & $5.49 \pm 0.29^{\#}$ & $10.68 \pm 1.00$ & $7.20 \pm 0.26^{\#}$ \\
ALT $(\mu \mathrm{kat} / \mathrm{l}) *$ & $1.19 \pm 0.05^{\#}$ & $1.01 \pm 0.12^{\#}$ & $0.78 \pm 0.02^{\#}$ & $0.08 \pm 0.15$ & $0.89 \pm 0.07^{\#}$ \\
AST $(\mu \mathrm{kat} / \mathrm{l})$ & $1.85 \pm 0.08$ & $1.97 \pm 0.22$ & $1.74 \pm 0.06$ & $1.36 \pm 0.40$ & $1.97 \pm 0.015$ \\
GMT $(\mu \mathrm{kat} / \mathrm{l}) *$ & $0.022 \pm 0.006^{\#}$ & $0.053 \pm 0.008^{\#}$ & $0.065 \pm 0.003^{\#}$ & $0.135 \pm 0.055$ & $0.098 \pm 0.020^{\#}$ \\
ALP $(\mu \mathrm{kat} / \mathrm{l}) *$ & $2.48 \pm 0.07^{\#}$ & $2.62 \pm 0.14^{\#}$ & $1.53 \pm 0.16$ & $0.87 \pm 0.05$ & $1.72 \pm 0.16^{\#}$ \\
Bilirubin $(\mu \mathrm{mol} / \mathrm{l}) *$ & $1.55 \pm 0.15^{\#}$ & $1.43 \pm 0.20^{\#}$ & $3.85 \pm 0.71^{\#}$ & $12.75 \pm 2.25$ & $3.92 \pm 0.47^{\#}$ \\
Urea $(\mathrm{mmol} / \mathrm{l}) *$ & $7.76 \pm 0.25^{\#}$ & $6.98 \pm 0.25^{\#}$ & $54.98 \pm 5.64^{\#}$ & $195.95 \pm 8.65$ & $48.92 \pm 5.35^{\#}$ \\
Creatinine $(\mu \mathrm{mol} / \mathrm{l}) *$ & $20.04 \pm 0.65^{\#}$ & $19.50 \pm 1.12^{\#}$ & $134.10 \pm 14.35^{\#}$ & $630.30 \pm 66.40$ & $119.77 \pm 13.19^{\#}$ \\
\hline
\end{tabular}

* denotes $\mathrm{P}<0.05$ statistically significant difference of one-way ANOVA; ${ }^{*}$ denotes statistical significance $\mathrm{P}<0.05$ of multiple comparisons versus SHR (FD + telmisartan) control group by Holm-Sidak method.

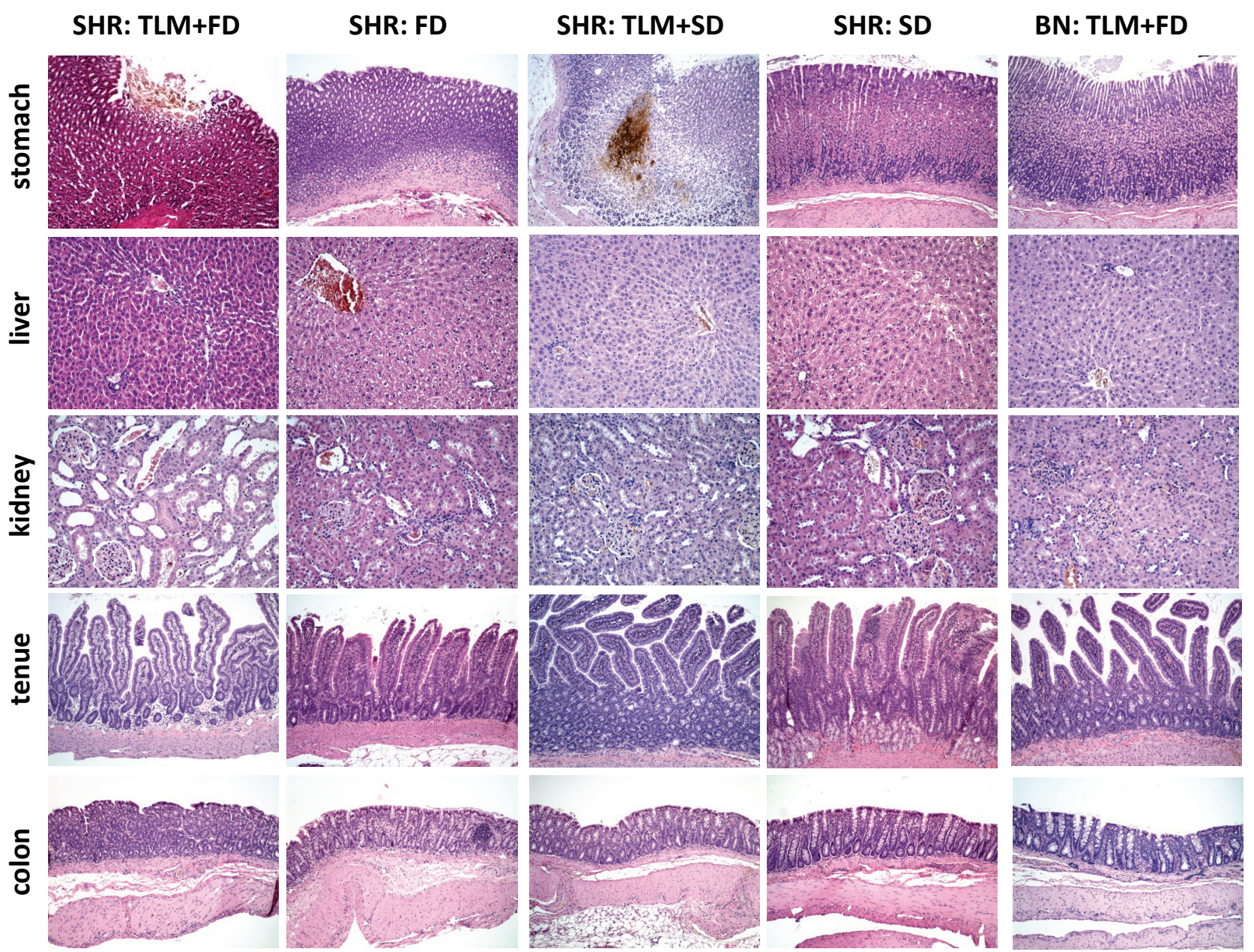

Fig. 3. Histopathological analysis. SHR rats exposed to telmisartan and high fructose diet displayed acute erosions and ulcerations of the upper gastrointestinal tract with bleeding. Additionally, morphology of acute tubular damage with dilation of proximal tubules and epithelial flattening and shedding was observed. No significant pathological changes were seen in liver, small intestine and colon in any group of animals. H\&E staining, original magnification x100. FD - fructose diet, SD - standard diet, TLM - telmisartan. 
Table 2. Telmisartan (TLM) concentrations in liver and plasma in SHR and BN strains fed a high fructose diet and treated with telmisartan for 13 days.

\begin{tabular}{lcccc}
\hline & \multicolumn{2}{c}{ SHR } & \multicolumn{2}{c}{ BN } \\
& TLM & TLM glucuronide & TLM & TLM glucuronide \\
\hline Liver $(\mu g / g)$ & & & \\
$T L M+S D$ & $26.0 \pm 2.9$ & $1.14 \pm 0.15$ & n.d. & n.d. \\
$T L M+F D$ & $25.1 \pm 3.8$ & $1.78 \pm 0.27$ & $6.4 \pm 0.9^{* *}$ & $0.38 \pm 0.03^{* *}$ \\
Plasma $(\mu \mathrm{g} / \mathrm{ml})$ & & & & \\
$T L M+S D$ & $18.7 \pm 2.3$ & $0.78 \pm 0.17$ & n.d. & n.d. \\
$T L M+F D$ & $19.5 \pm 4.6$ & $0.70 \pm 0.13$ & $3.9 \pm 0.8^{*}$ & $0.71 \pm 0.16$ \\
\hline
\end{tabular}

* and ** denote $\mathrm{P}<0.05$ and $\mathrm{P}<0.001$ statistically significant difference.

\section{Telmisartan concentrations in serum and livers}

As shown in Table 2, concentrations of both unconjugated telmisartan and telmisartan glucuronide in the liver of SHR rats fed a standard or high fructose diet were approximately 4 fold higher when compared to $\mathrm{BN}$ rats fed the same diet. Plasma concentrations of unconjugated telmisartan in the SHR were about 5 fold higher when compared to $\mathrm{BN}$ rats while levels of plasma telmisartan glucuronide were similar in both strains.

\section{Discussion}

In the current studies in SHR rats fed a high fructose diet, we observed acute toxic effects of clinically relevant doses of telmisartan characterized by weight loss, bleeding into the upper gastrointestinal tract, and death within 2-3 weeks of treatment. These pathological changes could be related to damage of the stomach or esophageal wall due to toxicity or allergic reaction (presence of eosinophil leukocytes), or due to tissue injury associated with a systemic stress response to hypotension.

The hepatic levels of telmisartan in SHR rats were significantly increased when compared to BN rats on both standard diet and high fructose diet. Thus, the SHR strain appears less efficient at excreting telmisartan via bile. The increased levels of telmisartan in treated SHR rats fed a standard chow were not toxic alone. The acute toxicity was observed only when treated SHR rats were fed a high fructose diet. It is possible that feeding a high fructose diet is associated with ATP depletion in the liver. ATP is necessary for normal function of ABC transporters that excrete conjugated telmisartan into bile.

A telmisartan dose of $5 \mathrm{mg} / \mathrm{kg} /$ day is usually well tolerated (Rabie et al. 2015, Shahataa et al. 2016). Toxicity studies in Wistar rats demonstrated that even a telmisartan dose of $150 \mathrm{mg} / \mathrm{kg} /$ day is not associated with any adverse effects (Sengupta et al. 2016). A single oral dose of telmisartan $2,000 \mathrm{mg} / \mathrm{kg}$ body weight also did not cause any apparent adverse effects in Wistar rats. However, intravenous administration of telmisartan at a dose of $250 \mathrm{mg} / \mathrm{kg} /$ day was reported to cause gastrointestinal ulcerations and mortality in Wistar rats (FDA Drug Approval Package website). We were able to find just 2 case reports in human patients treated with telmisartan that developed hemorrhage into the upper gastrointestinal tract (DrugInformer a and Patientsville.com websites). Thus similar adverse effects of telmisartan are very rare in humans. Telmisartan was also associated with a small but significant increase in risk for gastric ulcers (eHealthMe website). Relatively common adverse side effects include abdominal or stomach pain (DrugInformer b website). It is thus possible that some people are genetically predisposed to adverse side effects similar to those observe in SHR.

In conclusion, the results of the current studies suggest that the SHR is genetically predisposed to toxic effects of telmisartan when given in combination with a high fructose diet. These observations are consistent with the possibility that in some circumstances, SHR are predisposed to telmisartan toxicity possibly because of a genetically determined disturbance in telmisartan metabolism.

\section{Conflict of Interest}

There is no conflict of interest.

\section{Acknowledgements}

This work was supported by grant 14-36804G from the Grant Agency of the Czech Republic to M.P. 


\section{References}

BENSON SC, PERSHADSINGH HA, HO CI, CHITTIBOYINA A, DESAI P, PRAVENEC M, QI N, WANG J, AVERY MA, KURTZ TW: Identification of telmisartan as a unique angiotensin II receptor antagonist with selective PPARgamma-modulating activity. Hypertension 43: 993-1002, 2004.

DrugInformer a: www.druginformer.com/search/side_effect_details/Telmisartan/chronic\%20gastrointestinal\%20bleeding.html DrugInformer b: www.druginformer.com/search/side_effect_details/Telmisartan/abdominal\%20pain.html eHealthMe: www.ehealthme.com/ds/telmisartan/ulcer-stomach

FDA Drug Approval Package: http://www.accessdata.fda.gov/drugsatfda_docs/nda/98/20850_Micardis.cfm Patientsville.com: http://patientsville.com/telmisartan/gastrointestinal-haemorrhage.htm

PRAVENEC M, KŘEN V, LANDA V, MLEJNEK P, MUSILOVÁ A, ŠILHAVÝ J, ŠIMÁKOVÁ M, ZÍDEK V: Recent progress in the genetics of spontaneously hypertensive rats. Physiol Res 63 (Suppl 1): S1-S8, 2014.

RABIE EM, HEEBA GH, ABOUZIED MM, KHALIFA MM: Comparative effects of Aliskiren and Telmisartan in high fructose diet-induced metabolic syndrome in rats. Eur J Pharmacol 760: 145-153, 2015.

SENGUPTA P, DAS A, IBRAHIM F, MANDAL UK, CHATTERJEE B, MAHMOOD S, DAS SK, KIFAYATULLAH M: Safety profiling of pioglitazone and telmisartan combination by sub-chronic toxicity study in rat. Regul Toxicol Pharmacol 81: 155-161, 2016.

SHAHATAA MG, MOSTAFA-HEDEAB G, ALI EF, MAHDI EA, MAHMOUD FA: Effects of telmisartan and pioglitazone on high fructose induced metabolic syndrome in rats. Can J Physiol Pharmacol 94: 907-917, 2016.

YAN T, LI H, DENG L, GUO Y, YU W, FAWCETT JP, ZHANG D, CUI Y, GU J: Liquid chromatographic-tandem mass spectrometric method for the simultaneous quantitation of telmisartan and hydrochlorothiazide in human plasma. J Pharm Biomed Anal 48: 1225-1229, 2008. 